Article

\title{
Two Parametric Kinds of Eulerian-Type Polynomials Associated with Euler's Formula
}

\author{
Neslihan Kilar and Yilmaz Simsek * (D) \\ Department of Mathematics, Faculty of Science, University of Akdeniz, Antalya TR-07058, Turkey; \\ neslihankilar@gmail.com \\ * Correspondence: ysimsek@akdeniz.edu.tr
}

Received: 27 July 2019; Accepted: 16 August 2019; Published: 2 September 2019

\begin{abstract}
The purpose of this article is to construct generating functions for new families of special polynomials including two parametric kinds of Eulerian-type polynomials. Some fundamental properties of these functions are given. By using these generating functions and the Euler's formula, some identities and relations among trigonometric functions, two parametric kinds of Eulerian-type polynomials, Apostol-type polynomials, the Stirling numbers and Fubini-type polynomials are presented. Computational formulae for these polynomials are obtained. Applying a partial derivative operator to these generating functions, some derivative formulae and finite combinatorial sums involving the aforementioned polynomials and numbers are also obtained. In addition, some remarks and observations on these polynomials are given.
\end{abstract}

Keywords: Apostol-type numbers and polynomials; Stirling numbers; combinatorial sum; generating function; special functions

MSC: 05A15; 11B68; 11B73; 26C05; 33B10

\section{Introduction}

Special polynomials and their generating functions have important roles in many branches of mathematics, probability, statistics, mathematical physics and also engineering. Since polynomials are suitable for applying well-known operations such as derivative and integral, polynomials are very useful to study real-world problems in aforementioned areas. For instance, generating functions for special polynomials with their congruence properties, recurrence relations, computational formulae and symmetric sum involving these polynomials has been studied by many authors in recent years (cf. [1-27]).

In this article, by combining the Euler's formula with generating functions for two parametric kinds of Eulerian-type polynomials, their functional equations and partial derivative equations, we give many formulae and relations including the Stirling numbers, Fubini-type polynomials, two parametric kinds of Eulerian-type polynomials, and Apostol-type numbers and polynomials such as the Apostol-Bernoulli numbers and polynomials, the Apostol-Euler numbers and polynomials, and the Apostol-Genocchi numbers and polynomials.

Throughout this article, we use the following notations and definitions:

Let $\mathbb{N}=\{1,2,3, \ldots\}, \mathbb{N}_{0}=\mathbb{N} \cup\{0\}, \mathbb{Z}$ denote the set of integers, $\mathbb{R}$ denote the set of real numbers and $\mathbb{C}$ denote the set of complex numbers.

Furthermore, $0^{n}=1$ if $n=0$, and, $0^{n}=0$ if $n \in \mathbb{N}$, and $(\alpha)_{n}$ denotes the Pochhammer symbol, which is defined as follows:

$$
(\alpha)_{v}=\left\{\begin{array}{cr}
\alpha(\alpha+1)(\alpha+2) \ldots(\alpha+v-1) & v=n \in \mathbb{N}, \alpha \in \mathbb{C} . \\
1 & v=0, \alpha \in \mathbb{C} \backslash\{0\},
\end{array}\right.
$$


where $(0)_{0}=1$. Moreover,

$$
\left(\begin{array}{l}
\alpha \\
v
\end{array}\right)=\left\{\begin{array}{cc}
\frac{\alpha(\alpha-1)(\alpha-2) \ldots(\alpha-v+1)}{v !} & v \in \mathbb{N}, \alpha \in \mathbb{C} \\
1 & v=0
\end{array}\right.
$$

and

$$
(\alpha)^{\underline{v}}=(-1)^{v}(-\alpha)_{v}
$$

(cf. [1-25]).

The well-known Euler's formula is defined as follows:

$$
e^{i z}=\cos (z)+i \sin (z),
$$

where $i^{2}=-1$.

The Apostol-Bernoulli polynomials $\mathcal{B}_{n}^{(m)}(x ; \lambda)$ of order $m$ are defined by

$$
F_{A B}^{(m)}(t, x ; \lambda)=\left(\frac{t}{\lambda e^{t}-1}\right)^{m} e^{x t}=\sum_{n=0}^{\infty} \mathcal{B}_{n}^{(m)}(x ; \lambda) \frac{t^{n}}{n !},
$$

where $|t|<2 \pi$ when $\lambda=1 ;|t|<|\log (\lambda)|$ when $\lambda \neq 1$ and

$$
\mathcal{B}_{n}^{(m)}(\lambda)=\mathcal{B}_{n}^{(m)}(0 ; \lambda)
$$

where $\mathcal{B}_{n}^{(m)}(\lambda)$ denotes the so-called Apostol-Bernoulli numbers of order $m$ ( $\left.c f .[1,21,22,24,25]\right)$.

The Apostol-Euler polynomials $\mathcal{E}_{n}^{(m)}(x ; \lambda)$ of order $m$ are defined by

$$
F_{A E}^{(m)}(t, x ; \lambda)=\left(\frac{2}{\lambda e^{t}+1}\right)^{m} e^{x t}=\sum_{n=0}^{\infty} \mathcal{E}_{n}^{(m)}(x ; \lambda) \frac{t^{n}}{n !},
$$

where $|t|<\pi$ when $\lambda=1 ;|t|<|\log (-\lambda)|$ when $\lambda \neq 1$ and

$$
\mathcal{E}_{n}^{(m)}(\lambda)=\mathcal{E}_{n}^{(m)}(0 ; \lambda),
$$

where $\mathcal{E}_{n}^{(m)}(\lambda)$ denotes the so-called Apostol-Euler numbers of order $m(c f .[14,21-24])$.

The $\lambda$-Stirling numbers of the second kind are defined by

$$
F_{S 2}(t, m ; \lambda)=\frac{\left(\lambda e^{t}-1\right)^{m}}{m !}=\sum_{n=0}^{\infty} S_{2}(n, m ; \lambda) \frac{t^{n}}{n !},
$$

where $m \in \mathbb{N}_{0}$ and $\lambda \in \mathbb{C}(c f$. $[15,19,21])$. Substituting $\lambda=1$ into (3), the numbers $S_{2}(n, m ; \lambda)$ reduces to the Stirling numbers of the second kind:

$$
S_{2}(n, m)=S_{2}(n, m ; 1)
$$

(cf. [1-25]).

Combining (2) with (3), a computation formula for the Apostol-Euler polynomials of order $m$ is given as follows:

$$
\mathcal{E}_{n}^{(m)}(x ; \lambda)=2^{m} \sum_{k=0}^{n}\left(\begin{array}{l}
n \\
k
\end{array}\right) x^{n-k} \sum_{j=0}^{k}\left(\begin{array}{c}
m+j-1 \\
j
\end{array}\right) \frac{j !(-\lambda)^{j}}{(\lambda+1)^{j+m}} S_{2}(k, j)
$$

(cf. $[14,24])$. 
The Apostol-Genocchi polynomials $\mathcal{G}_{n}^{(m)}(x ; \lambda)$ of order $m$ are defined by

$$
F_{A G}^{(m)}(t, x ; \lambda)=\left(\frac{2 t}{\lambda e^{t}+1}\right)^{m} e^{x t}=\sum_{n=0}^{\infty} \mathcal{G}_{n}^{(m)}(x ; \lambda) \frac{t^{n}}{n !},
$$

where $|t|<\pi$ when $\lambda=1 ;|t|<|\log (-\lambda)|$ when $\lambda \neq 1$ and

$$
\mathcal{G}_{n}^{(m)}(\lambda)=\mathcal{G}_{n}^{(m)}(0 ; \lambda),
$$

where $\mathcal{G}_{n}^{(m)}(\lambda)$ denotes the so-called Apostol-Genocchi numbers of order $m(c f .[15,22,24])$.

The Apostol-type Frobenius-Euler polynomials $\mathcal{H}_{n}^{(m)}(x ; \lambda, u)$ of order $m$ are defined by

$$
F_{A H}^{(m)}(t, x ; \lambda, u)=\left(\frac{1-u}{\lambda e^{t}-u}\right)^{m} e^{x t}=\sum_{n=0}^{\infty} \mathcal{H}_{n}^{(m)}(x ; \lambda, u) \frac{t^{n}}{n !},
$$

where $u \in \mathbb{C}$ with $u \neq \lambda, \lambda \neq 1$ and

$$
\mathcal{H}_{n}^{(m)}(\lambda, u)=\mathcal{H}_{n}^{(m)}(0 ; \lambda, u)
$$

where $\mathcal{H}_{n}^{(m)}(\lambda, u)$ denotes the so-called Apostol-type Frobenius-Euler numbers of order $m$ (cf. $[2,19,22,24])$. Substituting $u=-1$ into (6), we have

$$
\mathcal{H}_{n}^{(m)}(x ; \lambda,-1)=\mathcal{E}_{n}^{(m)}(x ; \lambda)
$$

(cf. $[2,19,22,24])$.

Substituting $\lambda=1$ into (6), we have

$$
H_{n}^{(m)}(x ; u)=\mathcal{H}_{n}^{(m)}(x ; 1, u),
$$

where $H_{n}^{(m)}(x ; u)$ denotes the so-called Frobenius-Euler polynomials of order $m$.

Substituting $x=0$ into (7), we have

$$
H_{n}^{(m)}(0 ; u)=H_{n}^{(m)}(u),
$$

where $H_{n}^{(m)}(u)$ denotes the so-called Frobenius-Euler numbers of order $m$ ( $\left.c f .[4,5,10,12,13,17,19,20,22-24]\right)$.

By using (6) and (7), we have

$$
\mathcal{H}_{n}^{(m)}(x ; \lambda, u)=\left(\frac{1-u}{\lambda-u}\right)^{m} H_{n}^{(m)}\left(x ; \frac{u}{\lambda}\right) .
$$

The polynomials $C_{n}(x, y)$ and $S_{n}(x, y)$ are defined respectively by

$$
F_{C}(t, x, y)=e^{x t} \cos (y t)=\sum_{n=0}^{\infty} C_{n}(x, y) \frac{t^{n}}{n !}
$$

and

$$
F_{S}(t, x, y)=e^{x t} \sin (y t)=\sum_{n=0}^{\infty} S_{n}(x, y) \frac{t^{n}}{n !}
$$

$(c f .[11,16,25])$ 
By using (9) and (10), we have

$$
C_{n}(x, y)=\sum_{k=0}^{\left[\frac{n}{2}\right]}(-1)^{k}\left(\begin{array}{c}
n \\
2 k
\end{array}\right) x^{n-2 k} y^{2 k}
$$

and

$$
S_{n}(x, y)=\sum_{k=0}^{\left[\frac{n-1}{2}\right]}(-1)^{k}\left(\begin{array}{c}
n \\
2 k+1
\end{array}\right) x^{n-2 k-1} y^{2 k+1}
$$

(cf. $[11,16,25])$.

In [7], we defined the following generating function for the Fubini-type polynomials $a_{n}^{(m)}(x)$ of order $m$

$$
F_{a}^{(m)}(t, x)=\frac{2^{m}}{\left(2-e^{t}\right)^{2 m}} e^{x t}=\sum_{n=0}^{\infty} a_{n}^{(m)}(x) \frac{t^{n}}{n !} .
$$

Substituting $x=0$ into (11), we have

$$
a_{n}^{(m)}(0)=a_{n}^{(m)},
$$

where $a_{n}^{(m)}$ denotes the so-called Fubini-type numbers of order $m$ (cf. [7]).

In [9], we constructed the following generating functions for two kinds of Hermite-based $r$-parametric Milne-Thomson-type polynomials:

Let $r$-tuples $\vec{v}=\left(v_{1}, v_{2}, \ldots, v_{r}\right)$. Then, we have

$$
\begin{aligned}
B(t, x, y, z, \vec{v}, r, a, b) & =2(b+f(t, a))^{z} \exp (x t) M_{4}(t, y, \vec{v}, r) \\
& =\sum_{n=0}^{\infty} \mathfrak{h}_{1}(n, x, y, z ; \vec{v}, r, a, b) \frac{t^{n}}{n !},
\end{aligned}
$$

where

$$
M_{4}(t, y, \vec{v}, r)=\exp \left(\sum_{j=1}^{r} v_{j} t^{j}\right) \cos (y t)=\sum_{n=0}^{\infty} C_{n}(\vec{v}, y ; r) \frac{t^{n}}{n !}
$$

and

$$
\begin{aligned}
B_{1}(t, x, y, z, \vec{v}, r, a, b) & =2(b+f(t, a))^{z} \exp (x t) M_{5}(t, y, \vec{v}, r) \\
& =\sum_{n=0}^{\infty} \mathfrak{h}_{2}(n, x, y, z ; \vec{v}, r, a, b) \frac{t^{n}}{n !}
\end{aligned}
$$

where

$$
M_{5}(t, y, \vec{v}, r)=\exp \left(\sum_{j=1}^{r} v_{j} t^{j}\right) \sin (y t)=\sum_{n=0}^{\infty} S_{n}(\vec{v}, y ; r) \frac{t^{n}}{n !}
$$

The rest of this article is briefly summarized as follows:

In Section 2, we define generating functions for two parametric kinds of Eulerian-type polynomials. By using Euler's formula and these generating functions with their functional equations, we give relations and computation formulae for these polynomials. By using these formulae, we give a few values of these polynomials. Finally, we give some relations among the Apostol-Bernoulli polynomials, the Apostol-Euler polynomials, the Frobenius-Euler polynomials, the Apostol-Genocchi polynomials, the Stirling numbers, the Fubini-type polynomials and these polynomials.

In Section 3, we give functional equations and differential equations of these generating functions. By using these functional and differential equations, we derive derivative formulae and finite combinatorial sums involving the Apostol-Bernoulli numbers, the Apostol-Euler numbers, the Apostol-Genocchi numbers and for two parametric kinds of Eulerian-type polynomials. 
Section 4 is the conclusions section.

\section{New Families of Two Parametric Kinds of Eulerian-Type Polynomials}

In this section, we construct generating functions for two parametric kinds of Eulerian-type polynomials. By combining these functions with the Euler's formula, we give not only fundamental properties of these polynomials, but also new identities and relations related to the Apostol-Bernoulli numbers, the Apostol-Euler numbers, the Apostol-Genocchi numbers and for two parametric kinds of Eulerian-type polynomials.

We define the following generating functions for two parametric kinds of Eulerian-type polynomials:

$$
F_{H C}^{\left(k_{1}\right)}(t, x, y ; \lambda, u)=\left(\frac{1-u}{\lambda e^{t}-u}\right)^{k_{1}} e^{x t} \cos (y t)=\sum_{n=0}^{\infty} \mathcal{H}_{n}^{\left(C, k_{1}\right)}(x, y ; \lambda, u) \frac{t^{n}}{n !},
$$

and

$$
F_{H S}^{\left(k_{2}\right)}(t, x, y ; \lambda, u)=\left(\frac{1-u}{\lambda e^{t}-u}\right)^{k_{2}} e^{x t} \sin (y t)=\sum_{n=0}^{\infty} \mathcal{H}_{n}^{\left(S, k_{2}\right)}(x, y ; \lambda, u) \frac{t^{n}}{n !},
$$

where $k_{1}, k_{2} \in \mathbb{N}_{0}$.

The polynomials $\mathcal{H}_{n}^{\left(C, k_{1}\right)}(x, y ; \lambda, u)$ and $\mathcal{H}_{n}^{\left(S, k_{2}\right)}(x, y ; \lambda, u)$ are so-called two parametric kinds of Eulerian-type polynomials of order $k_{1}$ and $k_{2}$, respectively.

Note that the symbols $C$ and $S$ occurring in the superscripts on the right-hand sides of Equations (14) and (15) denote the trigonometric cosine and the trigonometric sine functions, respectively.

Remark 1. Substituting $b=0$ and

$$
f\left(t, \frac{a}{u}\right)=\left(\frac{u}{1-u}\right) \frac{1-u}{a e^{t}-u}
$$

and $\vec{v}=\overrightarrow{0}$ into Equations (12) and (13), we have the following identities, respectively:

$$
\mathfrak{h}_{1}\left(n, x, y, z ; \overrightarrow{0}, r, \frac{a}{u}, 0\right)=2\left(\frac{u}{1-u}\right)^{z} \mathcal{H}_{n}^{(C, z)}(x, y ; a, u)
$$

and

$$
\mathfrak{h}_{2}\left(n, x, y, z ; \overrightarrow{0}, r, \frac{a}{u}, 0\right)=2\left(\frac{u}{1-u}\right)^{z} \mathcal{H}_{n}^{(S, z)}(x, y ; a, u)
$$

Remark 2. Substituting $k_{1}=k_{2}=1$ into (14) and (15), we get the following generating functions, respectively:

$$
F_{H C}(t, x, y ; \lambda, u)=\frac{1-u}{\lambda e^{t}-u} e^{x t} \cos (y t)=\sum_{n=0}^{\infty} \mathcal{H}_{n}^{(C)}(x, y ; \lambda, u) \frac{t^{n}}{n !}
$$

and

$$
F_{H S}(t, x, y ; \lambda, u)=\frac{1-u}{\lambda e^{t}-u} e^{x t} \sin (y t)=\sum_{n=0}^{\infty} \mathcal{H}_{n}^{(S)}(x, y ; \lambda, u) \frac{t^{n}}{n !} .
$$

Remark 3. In ([19], p. 10), the second author defined following generating function for generalized Eulerian-type polynomials of order $m$ :

$$
F_{\lambda}^{(m)}(t, x ; u, a, b, c)=\left(\frac{a^{t}-u}{\lambda b^{t}-u}\right)^{m} c^{x t}=\sum_{n=0}^{\infty} \mathcal{H}_{n}^{(m)}(x ; u ; a, b, c ; \lambda) \frac{t^{n}}{n !} .
$$


Substituting $a=1, b=c=e$ into the above equation, we have

$$
F_{H C}^{\left(k_{1}\right)}(t, x, 0 ; \lambda, u)=F_{\lambda}^{\left(k_{1}\right)}(t, x ; u, 1, e, e)
$$

and

$$
F_{H S}^{\left(k_{2}\right)}\left(t, x, \frac{\pi}{2} ; \lambda, u\right)=F_{\lambda}^{\left(k_{2}\right)}(t, x ; u, 1, e, e) .
$$

Theorem 1. Let $n \in \mathbb{N}_{0}$. Then, we have

$$
\mathcal{H}_{n}^{(m)}(x+i y ; \lambda, u)=\mathcal{H}_{n}^{(C, m)}(x, y ; \lambda, u)+i \mathcal{H}_{n}^{(S, m)}(x, y ; \lambda, u) .
$$

Proof. By combining Equations (14) and (15) with the Euler's formula, we obtain

$$
\left(\frac{1-u}{\lambda e^{t}-u}\right)^{m} e^{(x+i y) t}=\sum_{n=0}^{\infty}\left(\mathcal{H}_{n}^{(C, m)}(x, y ; \lambda, u)+i \mathcal{H}_{n}^{(S, m)}(x, y ; \lambda, u)\right) \frac{t^{n}}{n !} .
$$

Therefore,

$$
\sum_{n=0}^{\infty} \mathcal{H}_{n}^{(m)}(x+i y ; \lambda, u) \frac{t^{n}}{n !}=\sum_{n=0}^{\infty}\left(\mathcal{H}_{n}^{(C, m)}(x, y ; \lambda, u)+i \mathcal{H}_{n}^{(S, m)}(x, y ; \lambda, u)\right) \frac{t^{n}}{n !} .
$$

Comparing the coefficients of $\frac{t^{n}}{n !}$ on both sides of the aforementioned equation, we arrive at the desired result.

Theorem 2. Let $n \in \mathbb{N}_{0}$. Then, we have

$$
C_{n}(x, y)=\left(\frac{u}{1-u}\right)^{k_{1}} k_{1} ! \sum_{j=0}^{n}\left(\begin{array}{l}
n \\
j
\end{array}\right) S_{2}\left(n-j, k_{1} ; \frac{\lambda}{u}\right) \mathcal{H}_{j}^{\left(C, k_{1}\right)}(x, y ; \lambda, u) .
$$

Proof. By using (3), (9) and (14), we get the following functional equation:

$$
u^{k_{1}} k_{1} ! F_{S 2}\left(t, k_{1} ; \frac{\lambda}{u}\right) F_{H C}^{\left(k_{1}\right)}(t, x, y ; \lambda, u)=(1-u)^{k_{1}} F_{C}(t, x, y) .
$$

Using the aforementioned equation, we get

$$
\left(\frac{1-u}{u}\right)^{k_{1}} \sum_{n=0}^{\infty} C_{n}(x, y) \frac{t^{n}}{n !}=k_{1} ! \sum_{n=0}^{\infty} S_{2}\left(n, k_{1} ; \frac{\lambda}{u}\right) \frac{t^{n}}{n !} \sum_{n=0}^{\infty} \mathcal{H}_{n}^{\left(C, k_{1}\right)}(x, y ; \lambda, u) \frac{t^{n}}{n !} .
$$

Therefore,

$$
\sum_{n=0}^{\infty} C_{n}(x, y) \frac{t^{n}}{n !}=\left(\frac{u}{1-u}\right)^{k_{1}} k_{1} ! \sum_{n=0}^{\infty} \sum_{j=0}^{n}\left(\begin{array}{c}
n \\
j
\end{array}\right) S_{2}\left(n-j, k_{1} ; \frac{\lambda}{u}\right) \mathcal{H}_{j}^{\left(C, k_{1}\right)}(x, y ; \lambda, u) \frac{t^{n}}{n !} .
$$

Comparing the coefficients of $\frac{t^{n}}{n !}$ on both sides of the aforementioned equation, we arrive at the desired result.

Theorem 3. Let $n \in \mathbb{N}_{0}$. Then, we have

$$
S_{n}(x, y)=\left(\frac{u}{1-u}\right)^{k_{2}} k_{2} ! \sum_{j=0}^{n}\left(\begin{array}{c}
n \\
j
\end{array}\right) S_{2}\left(n-j, k_{2} ; \frac{\lambda}{u}\right) \mathcal{H}_{j}^{\left(S, k_{2}\right)}(x, y ; \lambda, u) .
$$


Proof. By using (3), (10) and (15), we obtain the following functional equation:

$$
u^{k_{2}} k_{2} ! F_{S 2}\left(t, k_{2} ; \frac{\lambda}{u}\right) F_{H S}^{\left(k_{2}\right)}(t, x, y ; \lambda, u)=(1-u)^{k_{2}} F_{S}(t, x, y) .
$$

Using the aforementioned functional equation, we get

$$
\left(\frac{1-u}{u}\right)^{k_{2}} \sum_{n=0}^{\infty} S_{n}(x, y) \frac{t^{n}}{n !}=k_{2} ! \sum_{n=0}^{\infty} S_{2}\left(n, k_{2} ; \frac{\lambda}{u}\right) \frac{t^{n}}{n !} \sum_{n=0}^{\infty} \mathcal{H}_{n}^{\left(S, k_{2}\right)}(x, y ; \lambda, u) \frac{t^{n}}{n !} .
$$

Therefore,

$$
\sum_{n=0}^{\infty} S_{n}(x, y) \frac{t^{n}}{n !}=\left(\frac{u}{1-u}\right)^{k_{2}} k_{2} ! \sum_{n=0}^{\infty} \sum_{j=0}^{n}\left(\begin{array}{c}
n \\
j
\end{array}\right) S_{2}\left(n-j, k_{2} ; \frac{\lambda}{u}\right) \mathcal{H}_{j}^{\left(S, k_{2}\right)}(x, y ; \lambda, u) \frac{t^{n}}{n !} .
$$

Comparing the coefficients of $\frac{t^{n}}{n !}$ on both sides of the aforementioned equation, we arrive at the desired result.

Theorem 4. Let $n \in \mathbb{N}_{0}$. Then, we have

$$
\mathcal{H}_{n}^{\left(C, k_{1}\right)}(x, y ; \lambda, u)=\sum_{j=0}^{\left[\frac{n}{2}\right]}(-1)^{j}\left(\begin{array}{c}
n \\
2 j
\end{array}\right) \mathcal{H}_{n-2 j}^{\left(k_{1}\right)}(x ; \lambda, u) y^{2 j} .
$$

Proof. By using (14) and (6), we obtain the following functional equation:

$$
F_{H C}^{\left(k_{1}\right)}(t, x, y ; \lambda, u)=F_{A H}^{\left(k_{1}\right)}(t, x ; \lambda, u) \cos (y t) .
$$

By using the aforementioned equation, we get

$$
\sum_{n=0}^{\infty} \mathcal{H}_{n}^{\left(C, k_{1}\right)}(x, y ; \lambda, u) \frac{t^{n}}{n !}=\sum_{n=0}^{\infty} \mathcal{H}_{n}^{\left(k_{1}\right)}(x ; \lambda, u) \frac{t^{n}}{n !} \sum_{n=0}^{\infty}(-1)^{n} \frac{(y t)^{2 n}}{(2 n) !} .
$$

Therefore,

$$
\sum_{n=0}^{\infty} \mathcal{H}_{n}^{\left(C, k_{1}\right)}(x, y ; \lambda, u) \frac{t^{n}}{n !}=\sum_{n=0}^{\infty} \sum_{j=0}^{\left[\frac{n}{2}\right]}(-1)^{j}\left(\begin{array}{c}
n \\
2 j
\end{array}\right) \mathcal{H}_{n-2 j}^{\left(k_{1}\right)}(x ; \lambda, u) y^{2 j} \frac{t^{n}}{n !} .
$$

Comparing the coefficients of $\frac{t^{n}}{n !}$ on both sides of the aforementioned equation, we arrive at the desired result.

Combining (18) with (8), we get the following corollary:

Corollary 1. Let $n \in \mathbb{N}_{0}$. Then, we have

$$
\mathcal{H}_{n}^{\left(C, k_{1}\right)}(x, y ; \lambda, u)=\left(\frac{1-u}{\lambda-u}\right)^{k_{1}} \sum_{j=0}^{\left[\frac{n}{2}\right]}(-1)^{j}\left(\begin{array}{c}
n \\
2 j
\end{array}\right) y^{2 j} H_{n-2 j}^{\left(k_{1}\right)}\left(x ; \frac{u}{\lambda}\right) .
$$

Theorem 5. Let $n \in \mathbb{N}$. Then, we have

$$
\mathcal{H}_{n}^{\left(S, k_{2}\right)}(x, y ; \lambda, u)=\sum_{j=0}^{\left[\frac{n-1}{2}\right]}(-1)^{j}\left(\begin{array}{c}
n \\
2 j+1
\end{array}\right) \mathcal{H}_{n-1-2 j}^{\left(k_{2}\right)}(x ; \lambda, u) y^{2 j+1} .
$$


Proof. By using (15) and (6), we obtain the following functional equation:

$$
F_{H S}^{\left(k_{2}\right)}(t, x, y ; \lambda, u)=F_{A H}^{\left(k_{2}\right)}(t, x ; \lambda, u) \sin (y t)
$$

Using the aforementioned equation, we get

$$
\sum_{n=0}^{\infty} \mathcal{H}_{n}^{\left(S, k_{2}\right)}(x, y ; \lambda, u) \frac{t^{n}}{n !}=\sum_{n=0}^{\infty} \mathcal{H}_{n}^{\left(k_{2}\right)}(x ; \lambda, u) \frac{t^{n}}{n !} \sum_{n=0}^{\infty}(-1)^{n} \frac{(y t)^{2 n+1}}{(2 n+1) !} .
$$

Therefore,

$$
\sum_{n=0}^{\infty} \mathcal{H}_{n}^{\left(S, k_{2}\right)}(x, y ; \lambda, u) \frac{t^{n}}{n !}=\sum_{n=0}^{\infty} \sum_{j=0}^{\left[\frac{n-1}{2}\right]}(-1)^{j}\left(\begin{array}{c}
n \\
2 j+1
\end{array}\right) \mathcal{H}_{n-1-2 j}^{\left(k_{2}\right)}(x ; \lambda, u) y^{2 j+1} \frac{t^{n}}{n !}
$$

Comparing the coefficients of $\frac{t^{n}}{n !}$ on both sides of the aforementioned equation, we arrive at the desired result.

Combining (19) with (8), we arrive at the following corollary:

Corollary 2. Let $n \in \mathbb{N}$. Then, we have

$$
\mathcal{H}_{n}^{\left(S, k_{2}\right)}(x, y ; \lambda, u)=\left(\frac{1-u}{\lambda-u}\right)^{k_{2}} \sum_{j=0}^{\left[\frac{n-1}{2}\right]}(-1)^{j}\left(\begin{array}{c}
n \\
2 j+1
\end{array}\right) y^{2 j+1} H_{n-1-2 j}^{\left(k_{2}\right)}\left(x ; \frac{u}{\lambda}\right) .
$$

Theorem 6. Let $n \in \mathbb{N}_{0}$. Then, we have

$$
\mathcal{H}_{n}^{\left(C, k_{1}\right)}(x, y ; \lambda, u)=\left(\frac{1-u}{\lambda-u}\right)^{k_{1}} \sum_{j=0}^{n}\left(\begin{array}{c}
n \\
j
\end{array}\right) H_{j}^{\left(k_{1}\right)}\left(\frac{u}{\lambda}\right) C_{n-j}(x, y) .
$$

Proof. Using (8), (9) and (14), we get

$$
\sum_{n=0}^{\infty} \mathcal{H}_{n}^{\left(C, k_{1}\right)}(x, y ; \lambda, u) \frac{t^{n}}{n !}=\left(\frac{1-u}{\lambda-u}\right)^{k_{1}} \sum_{n=0}^{\infty} H_{n}^{\left(k_{1}\right)}\left(\frac{u}{\lambda}\right) \frac{t^{n}}{n !} \sum_{n=0}^{\infty} C_{n}(x, y) \frac{t^{n}}{n !} .
$$

Therefore,

$$
\sum_{n=0}^{\infty} \mathcal{H}_{n}^{\left(C, k_{1}\right)}(x, y ; \lambda, u) \frac{t^{n}}{n !}=\left(\frac{1-u}{\lambda-u}\right)^{k_{1}} \sum_{n=0}^{\infty} \sum_{j=0}^{n}\left(\begin{array}{c}
n \\
j
\end{array}\right) H_{j}^{\left(k_{1}\right)}\left(\frac{u}{\lambda}\right) C_{n-j}(x, y) \frac{t^{n}}{n !} .
$$

Comparing the coefficients of $\frac{t^{n}}{n !}$ on both sides of the aforementioned equation, we arrive at the desired result.

Theorem 7. Let $n \in \mathbb{N}_{0}$. Then, we have

$$
\mathcal{H}_{n}^{\left(S, k_{2}\right)}(x, y ; \lambda, u)=\left(\frac{1-u}{\lambda-u}\right)^{k_{2}} \sum_{j=0}^{n}\left(\begin{array}{c}
n \\
j
\end{array}\right) H_{j}^{\left(k_{2}\right)}\left(\frac{u}{\lambda}\right) S_{n-j}(x, y) .
$$

Proof. Using (8), (10) and (15), we obtain

$$
\sum_{n=0}^{\infty} \mathcal{H}_{n}^{\left(S, k_{2}\right)}(x, y ; \lambda, u) \frac{t^{n}}{n !}=\left(\frac{1-u}{\lambda-u}\right)^{k_{2}} \sum_{n=0}^{\infty} H_{n}^{\left(k_{2}\right)}\left(\frac{u}{\lambda}\right) \frac{t^{n}}{n !} \sum_{n=0}^{\infty} S_{n}(x, y) \frac{t^{n}}{n !} .
$$


Therefore,

$$
\sum_{n=0}^{\infty} \mathcal{H}_{n}^{\left(S, k_{2}\right)}(x, y ; \lambda, u) \frac{t^{n}}{n !}=\left(\frac{1-u}{\lambda-u}\right)^{k_{2}} \sum_{n=0}^{\infty} \sum_{j=0}^{n}\left(\begin{array}{c}
n \\
j
\end{array}\right) H_{j}^{\left(k_{2}\right)}\left(\frac{u}{\lambda}\right) S_{n-j}(x, y) \frac{t^{n}}{n !} .
$$

Comparing the coefficients of $\frac{t^{n}}{n !}$ on both sides of the aforementioned equation, we arrive at the desired result.

By using (20) and (21) in which $k_{1}=k_{2}=m$, we have

$$
\begin{aligned}
& \sum_{n=0}^{\infty}\left(\mathcal{H}_{n}^{(C, m)}(x, y ; \lambda, u)+i \mathcal{H}_{n}^{(S, m)}(x, y ; \lambda, u)\right) \frac{t^{n}}{n !} \\
= & \left(\frac{1-u}{\lambda-u}\right)^{m} \sum_{n=0}^{\infty} \sum_{j=0}^{n}\left(\begin{array}{c}
n \\
j
\end{array}\right) H_{j}^{(m)}\left(\frac{u}{\lambda}\right)\left(C_{n-j}(x, y)+i S_{n-j}(x, y)\right) \frac{t^{n}}{n !} .
\end{aligned}
$$

Using the aforementioned Equation (17) and Euler's formula, we obtain

$$
\sum_{n=0}^{\infty} \mathcal{H}_{n}^{(m)}(x+i y ; \lambda, u) \frac{t^{n}}{n !}=\left(\frac{1-u}{\lambda-u}\right)^{m} \sum_{n=0}^{\infty} \sum_{j=0}^{n}\left(\begin{array}{c}
n \\
j
\end{array}\right) H_{j}^{(m)}\left(\frac{u}{\lambda}\right)(x+i y)^{n-j} \frac{t^{n}}{n !} .
$$

Comparing the coefficients of $\frac{t^{n}}{n !}$ on both sides of the aforementioned equation, we get the following theorem:

Theorem 8. Let $n \in \mathbb{N}_{0}$. Then, we have

$$
\mathcal{H}_{n}^{(m)}(x+i y ; \lambda, u)=\left(\frac{1-u}{\lambda-u}\right)^{m} \sum_{j=0}^{n}\left(\begin{array}{l}
n \\
j
\end{array}\right) H_{j}^{(m)}\left(\frac{u}{\lambda}\right)(x+i y)^{n-j} .
$$

Theorem 9. Let $n \in \mathbb{N}_{0}$. Then, we have

$$
\mathcal{H}_{n}^{\left(S, k_{1}+k_{2}\right)}(2 x, 2 y ; \lambda, u)=2 \sum_{j=0}^{n}\left(\begin{array}{c}
n \\
j
\end{array}\right) \mathcal{H}_{j}^{\left(C, k_{1}\right)}(x, y ; \lambda, u) \mathcal{H}_{n-j}^{\left(S, k_{2}\right)}(x, y ; \lambda, u) .
$$

Proof. By using (14) and (15), we get the following functional equation:

$$
F_{H S}^{\left(k_{1}+k_{2}\right)}(t, 2 x, 2 y ; \lambda, u)=2 F_{H C}^{\left(k_{1}\right)}(t, x, y ; \lambda, u) F_{H S}^{\left(k_{2}\right)}(t, x, y ; \lambda, u)
$$

Using the aforementioned equation, we get

$$
\sum_{n=0}^{\infty} \mathcal{H}_{n}^{\left(S, k_{1}+k_{2}\right)}(2 x, 2 y ; \lambda, u) \frac{t^{n}}{n !}=2 \sum_{n=0}^{\infty} \mathcal{H}_{n}^{\left(C, k_{1}\right)}(x, y ; \lambda, u) \frac{t^{n}}{n !} \sum_{n=0}^{\infty} \mathcal{H}_{n}^{\left(S, k_{2}\right)}(x, y ; \lambda, u) \frac{t^{n}}{n !}
$$

Therefore,

$$
\sum_{n=0}^{\infty} \mathcal{H}_{n}^{\left(S, k_{1}+k_{2}\right)}(2 x, 2 y ; \lambda, u) \frac{t^{n}}{n !}=2 \sum_{n=0}^{\infty} \sum_{j=0}^{n}\left(\begin{array}{l}
n \\
j
\end{array}\right) \mathcal{H}_{j}^{\left(C, k_{1}\right)}(x, y ; \lambda, u) \mathcal{H}_{n-j}^{\left(S, k_{2}\right)}(x, y ; \lambda, u) \frac{t^{n}}{n !} .
$$

Comparing the coefficients of $\frac{t^{n}}{n !}$ on both sides of the aforementioned equation, we arrive at the desired result. 
Theorem 10. Let $n \in \mathbb{N}_{0}$. Then, we have

$$
2 \sum_{j=0}^{n}\left(\begin{array}{c}
n \\
j
\end{array}\right) \mathcal{H}_{j}^{\left(C, k_{1}\right)}(x, y ; \lambda, u) \mathcal{H}_{n-j}^{\left(S, k_{2}\right)}(x, y ; \lambda, u)=\sum_{j=0}^{n}\left(\begin{array}{l}
n \\
j
\end{array}\right) \mathcal{H}_{j}^{\left(k_{1}\right)}(x ; \lambda, u) \mathcal{H}_{n-j}^{\left(S, k_{2}\right)}(x, 2 y ; \lambda, u) .
$$

Proof. Combining (6), (14) with (15), we obtain the following functional equation:

$$
2 F_{H C}^{\left(k_{1}\right)}(t, x, y ; \lambda, u) F_{H S}^{\left(k_{2}\right)}(t, x, y ; \lambda, u)=F_{A H}^{\left(k_{1}\right)}(t, x ; \lambda, u) F_{H S}^{\left(k_{2}\right)}(t, x, 2 y ; \lambda, u) .
$$

From the above equation, we have

$$
2 \sum_{n=0}^{\infty} \mathcal{H}_{n}^{\left(C, k_{1}\right)}(x, y ; \lambda, u) \frac{t^{n}}{n !} \sum_{n=0}^{\infty} \mathcal{H}_{n}^{\left(S, k_{2}\right)}(x, y ; \lambda, u) \frac{t^{n}}{n !}=\sum_{n=0}^{\infty} \mathcal{H}_{n}^{\left(k_{1}\right)}(x ; \lambda, u) \frac{t^{n}}{n !} \sum_{n=0}^{\infty} \mathcal{H}_{n}^{\left(S, k_{2}\right)}(x, 2 y ; \lambda, u) \frac{t^{n}}{n !} .
$$

Therefore,

$2 \sum_{n=0}^{\infty} \sum_{j=0}^{n}\left(\begin{array}{l}n \\ j\end{array}\right) \mathcal{H}_{j}^{\left(C, k_{1}\right)}(x, y ; \lambda, u) \mathcal{H}_{n-j}^{\left(S, k_{2}\right)}(x, y ; \lambda, u) \frac{t^{n}}{n !}=\sum_{n=0}^{\infty} \sum_{j=0}^{n}\left(\begin{array}{c}n \\ j\end{array}\right) \mathcal{H}_{j}^{\left(k_{1}\right)}(x ; \lambda, u) \mathcal{H}_{n-j}^{\left(S, k_{2}\right)}(x, 2 y ; \lambda, u) \frac{t^{n}}{n !}$.

Comparing the coefficients of $\frac{t^{n}}{n !}$ on both sides of the aforementioned equation, we arrive at the desired result.

Theorem 11. Let $n \in \mathbb{N}_{0}$. Then, we have

$$
\mathcal{H}_{n-k_{1}}^{\left(C, k_{1}\right)}(x, y ; \lambda, u)=\left(\frac{1-u}{u}\right)^{k_{1}} \frac{1}{\left(\begin{array}{c}
n \\
k_{1}
\end{array}\right) k_{1} !} \sum_{j=0}^{\left[\frac{n}{2}\right]}(-1)^{j}\left(\begin{array}{c}
n \\
2 j
\end{array}\right) \mathcal{B}_{n-2 j}^{\left(k_{1}\right)}\left(x ; \frac{\lambda}{u}\right) y^{2 j} .
$$

Proof. Combining (1) with (14), we get the following functional equation:

$$
F_{H C}^{\left(k_{1}\right)}(t, x, y ; \lambda, u)=\left(\frac{1-u}{u t}\right)^{k_{1}} F_{A B}^{\left(k_{1}\right)}\left(t, x ; \frac{\lambda}{u}\right) \cos (y t) .
$$

By using the aforementioned equation, we get

$$
t^{k_{1}} \sum_{n=0}^{\infty} \mathcal{H}_{n}^{\left(C, k_{1}\right)}(x, y ; \lambda, u) \frac{t^{n}}{n !}=\left(\frac{1-u}{u}\right)^{k_{1}} \sum_{n=0}^{\infty} \mathcal{B}_{n}^{\left(k_{1}\right)}\left(x ; \frac{\lambda}{u}\right) \frac{t^{n}}{n !} \sum_{n=0}^{\infty}(-1)^{n} \frac{(y t)^{2 n}}{(2 n) !} .
$$

Therefore,

$$
\sum_{n=0}^{\infty}\left(\begin{array}{c}
n \\
k_{1}
\end{array}\right) k_{1} ! \mathcal{H}_{n-k_{1}}^{\left(C, k_{1}\right)}(x, y ; \lambda, u) \frac{t^{n}}{n !}=\left(\frac{1-u}{u}\right)^{k_{1}} \sum_{n=0}^{\infty} \sum_{j=0}^{\left[\frac{n}{2}\right]}(-1)^{j}\left(\begin{array}{c}
n \\
2 j
\end{array}\right) \mathcal{B}_{n-2 j}^{\left(k_{1}\right)}\left(x ; \frac{\lambda}{u}\right) y^{2 j} \frac{t^{n}}{n !} .
$$

Comparing the coefficients of $\frac{t^{n}}{n !}$ on both sides of the aforementioned equation, we arrive at the desired result.

Theorem 12. Let $n \in \mathbb{N}$. Then, we have

$$
\mathcal{H}_{n-k_{2}}^{\left(S, k_{2}\right)}(x, y ; \lambda, u)=\left(\frac{1-u}{u}\right)^{k_{2}} \frac{1}{\left(\begin{array}{c}
n \\
k_{2}
\end{array}\right) k_{2} !} \sum_{j=0}^{\left[\frac{n-1}{2}\right]}(-1)^{j}\left(\begin{array}{c}
n \\
2 j+1
\end{array}\right) \mathcal{B}_{n-1-2 j}^{\left(k_{2}\right)}\left(x ; \frac{\lambda}{u}\right) y^{2 j+1} .
$$


Proof. Combining (1) with (15), we obtain the following functional equation:

$$
F_{H S}^{\left(k_{2}\right)}(t, x, y ; \lambda, u)=\left(\frac{1-u}{u t}\right)^{k_{2}} F_{A B}^{\left(k_{2}\right)}\left(t, x ; \frac{\lambda}{u}\right) \sin (y t) .
$$

Using the above functional equation, observe that proof of the assertion of (25) follows precisely along the same lines as that proof of the assertion of (24), and so we omit it.

Theorem 13. Let $n \in \mathbb{N}_{0}$. Then, we have

$$
\mathcal{H}_{n-k_{1}}^{\left(C, k_{1}\right)}(x, y ; \lambda, u)=\left(\frac{u-1}{2 u}\right)^{k_{1}} \frac{1}{\left(\begin{array}{c}
n \\
k_{1}
\end{array}\right) k_{1} !} \sum_{j=0}^{\left[\frac{n}{2}\right]}(-1)^{j}\left(\begin{array}{c}
n \\
2 j
\end{array}\right) \mathcal{G}_{n-2 j}^{\left(k_{1}\right)}\left(x ;-\frac{\lambda}{u}\right) y^{2 j} .
$$

Proof. Combining (5) with (14), we have the following functional equation:

$$
F_{H C}^{\left(k_{1}\right)}(t, x, y ; \lambda, u)=\left(\frac{u-1}{2 u t}\right)^{k_{1}} F_{A G}^{\left(k_{1}\right)}\left(t, x ;-\frac{\lambda}{u}\right) \cos (y t) .
$$

Using the aforementioned equation, we get

$$
t^{k_{1}} \sum_{n=0}^{\infty} \mathcal{H}_{n}^{\left(C, k_{1}\right)}(x, y ; \lambda, u) \frac{t^{n}}{n !}=\left(\frac{u-1}{2 u}\right)^{k_{1}} \sum_{n=0}^{\infty} \mathcal{G}_{n}^{\left(k_{1}\right)}\left(x ;-\frac{\lambda}{u}\right) \frac{t^{n}}{n !} \sum_{n=0}^{\infty}(-1)^{n} \frac{(y t)^{2 n}}{(2 n) !} .
$$

Therefore,

$$
\sum_{n=0}^{\infty}\left(\begin{array}{c}
n \\
k_{1}
\end{array}\right) k_{1} ! \mathcal{H}_{n-k_{1}}^{\left(C, k_{1}\right)}(x, y ; \lambda, u) \frac{t^{n}}{n !}=\left(\frac{u-1}{2 u}\right)^{k_{1}} \sum_{n=0}^{\infty} \sum_{j=0}^{\left[\frac{n}{2}\right]}(-1)^{j}\left(\begin{array}{c}
n \\
2 j
\end{array}\right) \mathcal{G}_{n-2 j}^{\left(k_{1}\right)}\left(x ;-\frac{\lambda}{u}\right) y^{2 j} \frac{t^{n}}{n !} .
$$

Comparing the coefficients of $\frac{t^{n}}{n !}$ on both sides of the aforementioned equation, we arrive at the desired result.

Theorem 14. Let $n \in \mathbb{N}$. Then, we have

$$
\mathcal{H}_{n-k_{2}}^{\left(S, k_{2}\right)}(x, y ; \lambda, u)=\frac{\left(\frac{u-1}{2 u}\right)^{k_{2}}}{\left(\begin{array}{c}
n \\
k_{2}
\end{array}\right) k_{2} !} \sum_{j=0}^{\left[\frac{n-1}{2}\right]}(-1)^{j}\left(\begin{array}{c}
n \\
2 j+1
\end{array}\right) \mathcal{G}_{n-1-2 j}^{\left(k_{2}\right)}\left(x ;-\frac{\lambda}{u}\right) y^{2 j+1} .
$$

Proof. By using (5) and (15), we derive the following functional equation:

$$
F_{H S}^{\left(k_{2}\right)}(t, x, y ; \lambda, u)=\left(\frac{u-1}{2 u t}\right)^{k_{2}} F_{A G}^{\left(k_{2}\right)}\left(t, x ;-\frac{\lambda}{u}\right) \sin (y t) .
$$

From the above equation, observe that proof of the assertion of (27) follows precisely along the same lines as that proof of assertion of (26), and so we omit it.

Theorem 15. Let $n \in \mathbb{N}_{0}$. Then, we have

$$
\mathcal{H}_{n}^{\left(C, k_{1}\right)}(x, y ; \lambda, u)=\left(\frac{u-1}{2 u}\right)^{k_{1}\left[\frac{n}{2}\right]} \sum_{j=0}^{(-1)^{j}}\left(\begin{array}{c}
n \\
2 j
\end{array}\right) \mathcal{E}_{n-2 j}^{\left(k_{1}\right)}\left(x ;-\frac{\lambda}{u}\right) y^{2 j} .
$$


Proof. By using (2) and (14), we derive the following functional equation:

$$
F_{H C}^{\left(k_{1}\right)}(t, x, y ; \lambda, u)=\left(\frac{u-1}{2 u}\right)^{k_{1}} F_{A E}^{\left(k_{1}\right)}\left(t, x ;-\frac{\lambda}{u}\right) \cos (y t) .
$$

Using the aforementioned equation, we get

$$
\sum_{n=0}^{\infty} \mathcal{H}_{n}^{\left(C, k_{1}\right)}(x, y ; \lambda, u) \frac{t^{n}}{n !}=\left(\frac{u-1}{2 u}\right)^{k_{1}} \sum_{n=0}^{\infty} \mathcal{E}_{n}^{\left(k_{1}\right)}\left(x ;-\frac{\lambda}{u}\right) \frac{t^{n}}{n !} \sum_{n=0}^{\infty}(-1)^{n} \frac{(y t)^{2 n}}{(2 n) !} .
$$

Therefore,

$$
\sum_{n=0}^{\infty} \mathcal{H}_{n}^{\left(C, k_{1}\right)}(x, y ; \lambda, u) \frac{t^{n}}{n !}=\left(\frac{u-1}{2 u}\right)^{k_{1}} \sum_{n=0}^{\infty} \sum_{j=0}^{\left[\frac{n}{2}\right]}(-1)^{j}\left(\begin{array}{c}
n \\
2 j
\end{array}\right) \mathcal{E}_{n-2 j}^{\left(k_{1}\right)}\left(x ;-\frac{\lambda}{u}\right) y^{2 j} \frac{t^{n}}{n !}
$$

Comparing the coefficients of $\frac{t^{n}}{n !}$ on both sides of the aforementioned equation, we arrive at the desired result.

Combining (28) with (4), we have the following theorem:

Theorem 16. Let $n \in \mathbb{N}_{0}$. Then, we have

$$
\begin{aligned}
\mathcal{H}_{n}^{\left(C, k_{1}\right)}(x, y ; \lambda, u)= & \left(\frac{u-1}{u-\lambda}\right)^{k_{1}} \sum_{j=0}^{\left.\frac{n}{2}\right]}(-1)^{j}\left(\begin{array}{c}
n \\
2 j
\end{array}\right) y^{2 j} \\
& \times \sum_{l=0}^{n-2 j}\left(\begin{array}{c}
n-2 j \\
l
\end{array}\right) x^{n-2 j-l} \sum_{v=0}^{l}\left(\begin{array}{c}
k_{1}+v-1 \\
v
\end{array}\right) v !\left(\frac{\lambda}{u-\lambda}\right)^{v} S_{2}(l, v) .
\end{aligned}
$$

For $k_{1}=1,2,3$ and $n=1,2,3$, by using Equation (29), we compute a few values of the polynomials $\mathcal{H}_{n}^{\left(C, k_{1}\right)}(x, y ; \lambda, u)$ as follows:

For $k_{1}=1$, we have

$$
\begin{aligned}
\mathcal{H}_{0}^{(C, 1)}(x, y ; \lambda, u) & =\frac{u-1}{u-\lambda} \\
\mathcal{H}_{1}^{(C, 1)}(x, y ; \lambda, u) & =\frac{u-1}{u-\lambda}\left(\frac{\lambda}{u-\lambda}+x\right), \\
\mathcal{H}_{2}^{(C, 1)}(x, y ; \lambda, u) & =\frac{u-1}{u-\lambda}\left(\frac{2 \lambda^{2}}{(u-\lambda)^{2}}+\frac{\lambda(1+2 x)}{u-\lambda}+x^{2}-y^{2}\right), \\
\mathcal{H}_{3}^{(C, 1)}(x, y ; \lambda, u) & =\frac{u-1}{u-\lambda}\left(\frac{6 \lambda^{3}}{(u-\lambda)^{3}}+\frac{6 \lambda^{2}(1+x)}{(u-\lambda)^{2}}+\frac{\lambda\left(1+3 x+3 x^{2}-3 y^{2}\right)}{u-\lambda}+x^{3}-3 x y^{2}\right) .
\end{aligned}
$$

For $k_{1}=2$, we have

$$
\begin{aligned}
& \mathcal{H}_{0}^{(C, 2)}(x, y ; \lambda, u)=\left(\frac{u-1}{u-\lambda}\right)^{2} \\
& \mathcal{H}_{1}^{(C, 2)}(x, y ; \lambda, u)=\left(\frac{u-1}{u-\lambda}\right)^{2}\left(\frac{2 \lambda}{u-\lambda}+x\right) \\
& \mathcal{H}_{2}^{(C, 2)}(x, y ; \lambda, u)=\left(\frac{u-1}{u-\lambda}\right)^{2}\left(\frac{6 \lambda^{2}}{(u-\lambda)^{2}}+\frac{2 \lambda(1+2 x)}{u-\lambda}+x^{2}-y^{2}\right), \\
& \mathcal{H}_{3}^{(C, 2)}(x, y ; \lambda, u)=\left(\frac{u-1}{u-\lambda}\right)^{2}\left(\frac{24 \lambda^{3}}{(u-\lambda)^{3}}+\frac{18 \lambda^{2}(1+x)}{(u-\lambda)^{2}}+\frac{2 \lambda\left(1+3 x+3 x^{2}-3 y^{2}\right)}{u-\lambda}+x^{3}-3 x y^{2}\right) .
\end{aligned}
$$


For $k_{1}=3$, we have

$$
\begin{aligned}
& \mathcal{H}_{0}^{(C, 3)}(x, y ; \lambda, u)=\left(\frac{u-1}{u-\lambda}\right)^{3}, \\
& \mathcal{H}_{1}^{(C, 3)}(x, y ; \lambda, u)=\left(\frac{u-1}{u-\lambda}\right)^{3}\left(\frac{3 \lambda}{u-\lambda}+x\right), \\
& \mathcal{H}_{2}^{(C, 3)}(x, y ; \lambda, u)=\left(\frac{u-1}{u-\lambda}\right)^{3}\left(\frac{12 \lambda^{2}}{(u-\lambda)^{2}}+\frac{3 \lambda(1+2 x)}{u-\lambda}+x^{2}-y^{2}\right), \\
& \mathcal{H}_{3}^{(C, 3)}(x, y ; \lambda, u)=\left(\frac{u-1}{u-\lambda}\right)^{3}\left(\frac{60 \lambda^{3}}{(u-\lambda)^{3}}+\frac{36 \lambda^{2}(1+x)}{(u-\lambda)^{2}}+\frac{3 \lambda\left(1+3 x+3 x^{2}-3 y^{2}\right)}{u-\lambda}+x^{3}-3 x y^{2}\right) .
\end{aligned}
$$

Theorem 17. Let $n \in \mathbb{N}$. Then, we have

$$
\mathcal{H}_{n}^{\left(S, k_{2}\right)}(x, y ; \lambda, u)=\left(\frac{u-1}{2 u}\right)^{k_{2}\left[\frac{n-1}{2}\right]} \sum_{j=0}^{j}(-1)^{j}\left(\begin{array}{c}
n \\
2 j+1
\end{array}\right) \mathcal{E}_{n-1-2 j}^{\left(k_{2}\right)}\left(x ;-\frac{\lambda}{u}\right) y^{2 j+1} .
$$

Proof. Using (2) and (15), we derive the following functional equation:

$$
F_{H S}^{\left(k_{2}\right)}(t, x, y ; \lambda, u)=\left(\frac{u-1}{2 u}\right)^{k_{2}} F_{A E}^{\left(k_{2}\right)}\left(t, x ;-\frac{\lambda}{u}\right) \sin (y t) .
$$

From the above equation, observe that proof of the assertion of (30) follows precisely along the same lines as that proof of the assertion of (28), and so we omit it.

Combining (30) with (4), we have the following theorem:

Theorem 18. Let $n \in \mathbb{N}_{0}$. Then, we have

$$
\begin{aligned}
\mathcal{H}_{n}^{\left(S, k_{2}\right)}(x, y ; \lambda, u)= & \left(\frac{u-1}{u-\lambda}\right)^{k_{2}} \sum_{j=0}^{\left[\frac{n-1}{2}\right]}(-1)^{j}\left(\begin{array}{c}
n \\
2 j+1
\end{array}\right) y^{2 j+1} \\
& \times \sum_{l=0}^{n-1-2 j}\left(\begin{array}{c}
n-1-2 j \\
l
\end{array}\right) x^{n-1-2 j-l} \sum_{v=0}^{l}\left(\begin{array}{c}
k_{2}+v-1 \\
v
\end{array}\right) v !\left(\frac{\lambda}{u-\lambda}\right)^{v} S_{2}(l, v) .
\end{aligned}
$$

For $k_{2}=1,2,3$ and $n=1,2,3$, by using Equation (31), we compute a few values of the polynomials $\mathcal{H}_{n}^{\left(S, k_{2}\right)}(x, y ; \lambda, u)$ as follows:

For $k_{2}=1$, we have

$$
\begin{aligned}
\mathcal{H}_{0}^{(S, 1)}(x, y ; \lambda, u) & =0 \\
\mathcal{H}_{1}^{(S, 1)}(x, y ; \lambda, u) & =\frac{u-1}{u-\lambda} y, \\
\mathcal{H}_{2}^{(S, 1)}(x, y ; \lambda, u) & =\frac{u-1}{u-\lambda}\left(\frac{2 \lambda y}{u-\lambda}+2 x y\right) \\
\mathcal{H}_{3}^{(S, 1)}(x, y ; \lambda, u) & =\frac{u-1}{u-\lambda}\left(\frac{6 \lambda^{2} y}{(u-\lambda)^{2}}+\frac{3 \lambda(y+2 x y)}{u-\lambda}+3 x^{2} y-y^{3}\right) .
\end{aligned}
$$


For $k_{2}=2$, we have

$$
\begin{aligned}
\mathcal{H}_{0}^{(S, 2)}(x, y ; \lambda, u) & =0 \\
\mathcal{H}_{1}^{(S, 2)}(x, y ; \lambda, u) & =\left(\frac{u-1}{u-\lambda}\right)^{2} y, \\
\mathcal{H}_{2}^{(S, 2)}(x, y ; \lambda, u) & =\left(\frac{u-1}{u-\lambda}\right)^{2}\left(\frac{4 \lambda y}{u-\lambda}+2 x y\right), \\
\mathcal{H}_{3}^{(S, 2)}(x, y ; \lambda, u) & =\left(\frac{u-1}{u-\lambda}\right)^{2}\left(\frac{18 \lambda^{2} y}{(u-\lambda)^{2}}+\frac{6 \lambda(y+2 x y)}{u-\lambda}+3 x^{2} y-y^{3}\right) .
\end{aligned}
$$

For $k_{2}=3$, we have

$$
\begin{aligned}
& \mathcal{H}_{0}^{(S, 3)}(x, y ; \lambda, u)=0 \\
& \mathcal{H}_{1}^{(S, 3)}(x, y ; \lambda, u)=\left(\frac{u-1}{u-\lambda}\right)^{3} y, \\
& \mathcal{H}_{2}^{(S, 3)}(x, y ; \lambda, u)=\left(\frac{u-1}{u-\lambda}\right)^{3}\left(\frac{6 \lambda y}{u-\lambda}+2 x y\right), \\
& \mathcal{H}_{3}^{(S, 3)}(x, y ; \lambda, u)=\left(\frac{u-1}{u-\lambda}\right)^{3}\left(\frac{36 \lambda^{2} y}{(u-\lambda)^{2}}+\frac{9 \lambda(y+2 x y)}{u-\lambda}+3 x^{2} y-y^{3}\right) .
\end{aligned}
$$

Theorem 19. Let $n \in \mathbb{N}_{0}$. Then, we have

$$
H_{n}^{\left(C, 2 k_{1}\right)}(x, y ; 1,2)=\frac{1}{2^{k_{1}}} \sum_{j=0}^{\left[\frac{n}{2}\right]}(-1)^{j}\left(\begin{array}{c}
n \\
2 j
\end{array}\right) a_{n-2 j}^{\left(k_{1}\right)}(x) y^{2 j} .
$$

Proof. By using (11) and (14), the following functional equation is obtained:

$$
F_{H C}^{\left(2 k_{1}\right)}(t, x, y ; 1,2)=\frac{1}{2^{k_{1}}} F_{a}^{\left(k_{1}\right)}(t, x) \cos (y t) .
$$

Using the aforementioned equation, we get

$$
\sum_{n=0}^{\infty} H_{n}^{\left(C, 2 k_{1}\right)}(x, y ; 1,2) \frac{t^{n}}{n !}=\frac{1}{2^{k_{1}}} \sum_{n=0}^{\infty} \sum_{j=0}^{\left[\frac{n}{2}\right]}(-1)^{j}\left(\begin{array}{c}
n \\
2 j
\end{array}\right) a_{n-2 j}^{\left(k_{1}\right)}(x) y^{2 j} \frac{t^{n}}{n !} .
$$

Comparing the coefficients of $\frac{t^{n}}{n !}$ on both sides of the aforementioned equation, we arrive at the desired result.

Theorem 20. Let $n \in \mathbb{N}$. Then, we have

$$
H_{n}^{\left(S, 2 k_{2}\right)}(x, y ; 1,2)=\frac{1}{2^{k_{2}}} \sum_{j=0}^{\left[\frac{n-1}{2}\right]}(-1)^{j}\left(\begin{array}{c}
n \\
2 j+1
\end{array}\right) a_{n-1-2 j}^{\left(k_{2}\right)}(x) y^{2 j+1} .
$$

Proof. By using (11) and (15), we derive the following functional equation:

$$
F_{H S}^{\left(2 k_{2}\right)}(t, x, y ; 1,2)=\frac{1}{2^{k_{2}}} F_{a}^{\left(k_{2}\right)}(t, x) \sin (y t) .
$$

From the above equation, observe that proof of the assertion of (33) follows precisely along the same lines as that proof of the assertion of (32), and so we omit it. 


\section{Partial Derivative Equations of Generating Functions for Two Parametric Kinds of Eulerian-Type Polynomials}

In this section, by applying partial derivative operator to Equations (14) and (15), we give some derivative formulae and finite combinatorial sums for the two parametric kinds of Eulerian-type polynomials.

\subsection{Partial Derivative Formulas for the Polynomials $\mathcal{H}_{n}^{\left(C, k_{1}\right)}(x, y ; \lambda, u)$}

Here, partial derivative equations of generating functions for the polynomials $\mathcal{H}_{n}^{\left(C, k_{1}\right)}(x, y ; \lambda, u)$ are given. By applying partial differential operator to Equation (14), we derive derivative formulae and finite combinatorial sums related to the polynomials $\mathcal{H}_{n}^{\left(C, k_{1}\right)}(x, y ; \lambda, u)$, the Apostol-Bernoulli numbers of higher order, the Apostol-Euler numbers of higher order, and the Apostol-Genocchi numbers of higher order.

Theorem 21. Let $n, m \in \mathbb{N}$. Then, we have

$$
\frac{\partial^{m}}{\partial x^{m}}\left\{\mathcal{H}_{n}^{\left(C, k_{1}\right)}(x, y ; \lambda, u)\right\}=\left(\frac{1-u}{u}\right)^{m} \sum_{j=0}^{n}\left(\begin{array}{c}
n \\
j
\end{array}\right) \mathcal{B}_{j}^{(m)}\left(\frac{\lambda}{u}\right) \mathcal{H}_{n-j}^{\left(C, k_{1}-m\right)}(x, y ; \lambda, u) .
$$

Proof. By applying the derivative operator $\frac{\partial^{m}}{\partial x^{m}}$ to Equation (14), we get

$$
\sum_{n=0}^{\infty} \frac{\partial^{m}}{\partial x^{m}}\left\{\mathcal{H}_{n}^{\left(C, k_{1}\right)}(x, y ; \lambda, u)\right\} \frac{t^{n}}{n !}=\left(\frac{1-u}{u}\right)^{m} \sum_{n=0}^{\infty} \mathcal{B}_{n}^{(m)}\left(\frac{\lambda}{u}\right) \frac{t^{n}}{n !} \sum_{n=0}^{\infty} \mathcal{H}_{n}^{\left(C, k_{1}-m\right)}(x, y ; \lambda, u) \frac{t^{n}}{n !} .
$$

Therefore,

$$
\sum_{n=0}^{\infty} \frac{\partial^{m}}{\partial x^{m}}\left\{\mathcal{H}_{n}^{\left(C, k_{1}\right)}(x, y ; \lambda, u)\right\} \frac{t^{n}}{n !}=\left(\frac{1-u}{u}\right)^{m} \sum_{n=0}^{\infty} \sum_{j=0}^{n}\left(\begin{array}{c}
n \\
j
\end{array}\right) \mathcal{B}_{j}^{(m)}\left(\frac{\lambda}{u}\right) \mathcal{H}_{n-j}^{\left(C, k_{1}-m\right)}(x, y ; \lambda, u) \frac{t^{n}}{n !} .
$$

Comparing the coefficients of $\frac{t^{n}}{n !}$ on both sides of the aforementioned equation, we arrive at the desired result.

Theorem 22. Let $n, m \in \mathbb{N}$. Then, we have

$$
\frac{\partial^{m}}{\partial x^{m}}\left\{\mathcal{H}_{n}^{\left(C, k_{1}\right)}(x, y ; \lambda, u)\right\}=\left(\frac{u-1}{2 u}\right)^{m} \sum_{j=0}^{n}\left(\begin{array}{c}
n \\
j
\end{array}\right) \mathcal{G}_{j}^{(m)}\left(-\frac{\lambda}{u}\right) \mathcal{H}_{n-j}^{\left(C, k_{1}-m\right)}(x, y ; \lambda, u)
$$

Proof. By applying the derivative operator $\frac{\partial^{m}}{\partial x^{m}}$ to Equation (14), we get

$\sum_{n=0}^{\infty} \frac{\partial^{m}}{\partial x^{m}}\left\{\mathcal{H}_{n}^{\left(C, k_{1}\right)}(x, y ; \lambda, u)\right\} \frac{t^{n}}{n !}=\left(\frac{u-1}{2 u}\right)^{m} \sum_{n=0}^{\infty} \mathcal{G}_{n}^{(m)}\left(-\frac{\lambda}{u}\right) \frac{t^{n}}{n !} \sum_{n=0}^{\infty} \mathcal{H}_{n}^{\left(C, k_{1}-m\right)}(x, y ; \lambda, u) \frac{t^{n}}{n !}$.

Therefore,

$$
\sum_{n=0}^{\infty} \frac{\partial^{m}}{\partial x^{m}}\left\{\mathcal{H}_{n}^{\left(C, k_{1}\right)}(x, y ; \lambda, u)\right\} \frac{t^{n}}{n !}=\left(\frac{u-1}{2 u}\right)^{m} \sum_{n=0}^{\infty} \sum_{j=0}^{n}\left(\begin{array}{l}
n \\
j
\end{array}\right) \mathcal{G}_{j}^{(m)}\left(-\frac{\lambda}{u}\right) \mathcal{H}_{n-j}^{\left(C, k_{1}-m\right)}(x, y ; \lambda, u) \frac{t^{n}}{n !} .
$$

Comparing the coefficients of $\frac{t^{n}}{n !}$ on both sides of the aforementioned equation, we arrive at the desired result. 
Theorem 23. Let $n, m \in \mathbb{N}$ with $n \geq m$. Then, we have

$$
\frac{\partial^{m}}{\partial x^{m}}\left\{\mathcal{H}_{n}^{\left(C, k_{1}\right)}(x, y ; \lambda, u)\right\}=\left(\frac{u-1}{2 u}\right)^{m}\left(\begin{array}{c}
n \\
m
\end{array}\right) m ! \sum_{j=0}^{n-m}\left(\begin{array}{c}
n-m \\
j
\end{array}\right) \mathcal{E}_{j}^{(m)}\left(-\frac{\lambda}{u}\right) \mathcal{H}_{n-m-j}^{\left(C, k_{1}-m\right)}(x, y ; \lambda, u) .
$$

Proof. By applying the derivative operator $\frac{\partial^{m}}{\partial x^{m}}$ to Equation (14), we obtain

$$
\sum_{n=0}^{\infty} \frac{\partial^{m}}{\partial x^{m}}\left\{\mathcal{H}_{n}^{\left(C, k_{1}\right)}(x, y ; \lambda, u)\right\} \frac{t^{n}}{n !}=\left(\frac{u-1}{2 u}\right)^{m} t^{m} \sum_{n=0}^{\infty} \mathcal{E}_{n}^{(m)}\left(-\frac{\lambda}{u}\right) \frac{t^{n}}{n !} \sum_{n=0}^{\infty} \mathcal{H}_{n}^{\left(C, k_{1}-m\right)}(x, y ; \lambda, u) \frac{t^{n}}{n !}
$$

Therefore,

$$
\begin{aligned}
& \sum_{n=0}^{\infty} \frac{\partial^{m}}{\partial x^{m}}\left\{\mathcal{H}_{n}^{\left(C, k_{1}\right)}(x, y ; \lambda, u)\right\} \frac{t^{n}}{n !} \\
= & \left(\frac{u-1}{2 u}\right)^{m} \sum_{n=0}^{\infty} \sum_{j=0}^{n-m}\left(\begin{array}{c}
n-m \\
j
\end{array}\right)\left(\begin{array}{c}
n \\
m
\end{array}\right) m ! \mathcal{E}_{j}^{(m)}\left(-\frac{\lambda}{u}\right) \mathcal{H}_{n-m-j}^{\left(C, k_{1}-m\right)}(x, y ; \lambda, u) \frac{t^{n}}{n !} .
\end{aligned}
$$

Comparing the coefficients of $\frac{t^{n}}{n !}$ on both sides of the aforementioned equation, we arrive at the desired result.

Combining (34) and (35) with (36), we arrive at the following theorem:

Theorem 24. Let $n, m \in \mathbb{N}_{0}$ with $n \geq m$. Then, we have

$$
\begin{gathered}
\sum_{j=0}^{n}\left(\begin{array}{c}
n \\
j
\end{array}\right) \mathcal{B}_{j}^{(m)}\left(\frac{\lambda}{u}\right) \mathcal{H}_{n-j}^{\left(C, k_{1}-m\right)}(x, y ; \lambda, u)=\frac{m !}{(-2)^{m}}\left(\begin{array}{c}
n \\
m
\end{array}\right) \sum_{j=0}^{n-m}\left(\begin{array}{c}
n-m \\
j
\end{array}\right) \mathcal{E}_{j}^{(m)}\left(-\frac{\lambda}{u}\right) \mathcal{H}_{n-m-j}^{\left(C, k_{1}-m\right)}(x, y ; \lambda, u), \\
\sum_{j=0}^{n}\left(\begin{array}{c}
n \\
j
\end{array}\right) \mathcal{B}_{j}^{(m)}\left(\frac{\lambda}{u}\right) \mathcal{H}_{n-j}^{\left(C, k_{1}-m\right)}(x, y ; \lambda, u)=\frac{1}{(-2)^{m}} \sum_{j=0}^{n}\left(\begin{array}{c}
n \\
j
\end{array}\right) \mathcal{G}_{j}^{(m)}\left(-\frac{\lambda}{u}\right) \mathcal{H}_{n-j}^{\left(C, k_{1}-m\right)}(x, y ; \lambda, u),
\end{gathered}
$$

and

$$
\sum_{j=0}^{n}\left(\begin{array}{c}
n \\
j
\end{array}\right) \mathcal{G}_{j}^{(m)}\left(-\frac{\lambda}{u}\right) \mathcal{H}_{n-j}^{\left(C, k_{1}-m\right)}(x, y ; \lambda, u)=\left(\begin{array}{c}
n \\
m
\end{array}\right) m ! \sum_{j=0}^{n-m}\left(\begin{array}{c}
n-m \\
j
\end{array}\right) \mathcal{E}_{j}^{(m)}\left(-\frac{\lambda}{u}\right) \mathcal{H}_{n-m-j}^{\left(C, k_{1}-m\right)}(x, y ; \lambda, u) .
$$

3.2. Partial Derivative Formulas for the Polynomials $\mathcal{H}_{n}^{\left(S, k_{2}\right)}(x, y ; \lambda, u)$

Here, partial derivative equations for generating functions of the polynomials $\mathcal{H}_{n}^{\left(S, k_{2}\right)}(x, y ; \lambda, u)$ are given.

By applying the partial differential operator to Equation (15), we give derivative formulae and finite combinatorial sums related to the polynomials $\mathcal{H}_{n}^{\left(S, k_{2}\right)}(x, y ; \lambda, u)$, the Apostol-Bernoulli numbers of higher order, the Apostol-Euler numbers of higher order, and the Apostol-Genocchi numbers of higher order.

Theorem 25. Let $n, m \in \mathbb{N}$. Then, we have

$$
\frac{\partial^{m}}{\partial x^{m}}\left\{\mathcal{H}_{n}^{\left(S, k_{2}\right)}(x, y ; \lambda, u)\right\}=\left(\frac{1-u}{u}\right)^{m} \sum_{j=0}^{n}\left(\begin{array}{c}
n \\
j
\end{array}\right) \mathcal{B}_{j}^{(m)}\left(\frac{\lambda}{u}\right) \mathcal{H}_{n-j}^{\left(S, k_{2}-m\right)}(x, y ; \lambda, u) .
$$


Proof. By applying the derivative operator $\frac{\partial^{m}}{\partial x^{m}}$ to Equation (15), we have

$$
\sum_{n=0}^{\infty} \frac{\partial^{m}}{\partial x^{m}}\left\{\mathcal{H}_{n}^{\left(S, k_{2}\right)}(x, y ; \lambda, u)\right\} \frac{t^{n}}{n !}=\left(\frac{1-u}{u}\right)^{m} \sum_{n=0}^{\infty} \mathcal{B}_{n}^{(m)}\left(\frac{\lambda}{u}\right) \frac{t^{n}}{n !} \sum_{n=0}^{\infty} \mathcal{H}_{n}^{\left(S, k_{2}-m\right)}(x, y ; \lambda, u) \frac{t^{n}}{n !} .
$$

Therefore,

$$
\sum_{n=0}^{\infty} \frac{\partial^{m}}{\partial x^{m}}\left\{\mathcal{H}_{n}^{\left(S, k_{2}\right)}(x, y ; \lambda, u)\right\} \frac{t^{n}}{n !}=\left(\frac{1-u}{u}\right)^{m} \sum_{n=0}^{\infty} \sum_{j=0}^{n}\left(\begin{array}{c}
n \\
j
\end{array}\right) \mathcal{B}_{j}^{(m)}\left(\frac{\lambda}{u}\right) \mathcal{H}_{n-j}^{\left(S, k_{2}-m\right)}(x, y ; \lambda, u) \frac{t^{n}}{n !} .
$$

Comparing the coefficients of $\frac{t^{n}}{n !}$ on both sides of the aforementioned equation, we arrive at the desired result.

Theorem 26. Let $n, m \in \mathbb{N}$. Then, we have

$$
\frac{\partial^{m}}{\partial x^{m}}\left\{\mathcal{H}_{n}^{\left(S, k_{2}\right)}(x, y ; \lambda, u)\right\}=\left(\frac{u-1}{2 u}\right)^{m} \sum_{j=0}^{n}\left(\begin{array}{c}
n \\
j
\end{array}\right) \mathcal{G}_{j}^{(m)}\left(-\frac{\lambda}{u}\right) \mathcal{H}_{n-j}^{\left(S, k_{2}-m\right)}(x, y ; \lambda, u) .
$$

Proof. By applying the derivative operator $\frac{\partial^{m}}{\partial x^{m}}$ to Equation (15), we get

$$
\sum_{n=0}^{\infty} \frac{\partial^{m}}{\partial x^{m}}\left\{\mathcal{H}_{n}^{\left(S, k_{2}\right)}(x, y ; \lambda, u)\right\} \frac{t^{n}}{n !}=\left(\frac{1-u}{-2 u}\right)^{m} \sum_{n=0}^{\infty} \mathcal{G}_{n}^{(m)}\left(-\frac{\lambda}{u}\right) \frac{t^{n}}{n !} \sum_{n=0}^{\infty} \mathcal{H}_{n}^{\left(S, k_{2}-m\right)}(x, y ; \lambda, u) \frac{t^{n}}{n !} .
$$

Therefore,

$$
\sum_{n=0}^{\infty} \frac{\partial^{m}}{\partial x^{m}}\left\{\mathcal{H}_{n}^{\left(S, k_{2}\right)}(x, y ; \lambda, u)\right\} \frac{t^{n}}{n !}=\left(\frac{1-u}{-2 u}\right)^{m} \sum_{n=0}^{\infty} \sum_{j=0}^{n}\left(\begin{array}{c}
n \\
j
\end{array}\right) \mathcal{G}_{j}^{(m)}\left(-\frac{\lambda}{u}\right) \mathcal{H}_{n-j}^{\left(S, k_{2}-m\right)}(x, y ; \lambda, u) \frac{t^{n}}{n !} .
$$

Comparing the coefficients of $\frac{t^{n}}{n !}$ on both sides of the aforementioned equation, after some elementary calculations, we arrive at the desired result.

Theorem 27. Let $n, m \in \mathbb{N}$ with $n \geq m$. Then, we have

$$
\frac{\partial^{m}}{\partial x^{m}}\left\{\mathcal{H}_{n}^{\left(S, k_{2}\right)}(x, y ; \lambda, u)\right\}=\left(\frac{u-1}{2 u}\right)^{m}\left(\begin{array}{c}
n \\
m
\end{array}\right) m ! \sum_{j=0}^{n-m}\left(\begin{array}{c}
n-m \\
j
\end{array}\right) \mathcal{E}_{j}^{(m)}\left(-\frac{\lambda}{u}\right) \mathcal{H}_{n-m-j}^{\left(S, k_{2}-m\right)}(x, y ; \lambda, u) .
$$

Proof. By applying derivative operator $\frac{\partial^{m}}{\partial x^{m}}$ to Equation (15), we obtain

$$
\sum_{n=0}^{\infty} \frac{\partial^{m}}{\partial x^{m}}\left\{\mathcal{H}_{n}^{\left(S, k_{2}\right)}(x, y ; \lambda, u)\right\} \frac{t^{n}}{n !}=\left(\frac{1-u}{-2 u}\right)^{m} t^{m} \sum_{n=0}^{\infty} \mathcal{E}_{n}^{(m)}\left(-\frac{\lambda}{u}\right) \frac{t^{n}}{n !} \sum_{n=0}^{\infty} \mathcal{H}_{n}^{\left(S, k_{2}-m\right)}(x, y ; \lambda, u) \frac{t^{n}}{n !}
$$

Therefore,

$$
\begin{aligned}
& \sum_{n=0}^{\infty} \frac{\partial^{m}}{\partial x^{m}}\left\{\mathcal{H}_{n}^{\left(S, k_{2}\right)}(x, y ; \lambda, u)\right\} \frac{t^{n}}{n !} \\
= & \left(\frac{1-u}{-2 u}\right)^{m} \sum_{n=0}^{\infty}\left(\begin{array}{c}
n \\
m
\end{array}\right) m ! \sum_{j=0}^{n-m}\left(\begin{array}{c}
n-m \\
j
\end{array}\right) \mathcal{E}_{j}^{(m)}\left(-\frac{\lambda}{u}\right) \mathcal{H}_{n-m-j}^{\left(S, k_{2}-m\right)}(x, y ; \lambda, u) \frac{t^{n}}{n !} .
\end{aligned}
$$

Comparing the coefficients of $\frac{t^{n}}{n !}$ on both sides of the aforementioned equation, after some elementary calculations, we arrive at the desired result.

Combining (37) and (38) with (39), we arrive at the following theorem: 
Theorem 28. Let $n, m \in \mathbb{N}_{0}$ with $n \geq m$. Then, we have

$$
\begin{gathered}
\sum_{j=0}^{n}\left(\begin{array}{c}
n \\
j
\end{array}\right) \mathcal{B}_{j}^{(m)}\left(\frac{\lambda}{u}\right) \mathcal{H}_{n-j}^{\left(S, k_{2}-m\right)}(x, y ; \lambda, u)=\frac{m !}{(-2)^{m}}\left(\begin{array}{c}
n \\
m
\end{array}\right) \sum_{j=0}^{n-m}\left(\begin{array}{c}
n-m \\
j
\end{array}\right) \mathcal{E}_{j}^{(m)}\left(-\frac{\lambda}{u}\right) \mathcal{H}_{n-m-j}^{\left(S, k_{2}-m\right)}(x, y ; \lambda, u), \\
\sum_{j=0}^{n}\left(\begin{array}{c}
n \\
j
\end{array}\right) \mathcal{B}_{j}^{(m)}\left(\frac{\lambda}{u}\right) \mathcal{H}_{n-j}^{\left(S, k_{2}-m\right)}(x, y ; \lambda, u)=\frac{1}{(-2)^{m}} \sum_{j=0}^{n}\left(\begin{array}{c}
n \\
j
\end{array}\right) \mathcal{G}_{j}^{(m)}\left(-\frac{\lambda}{u}\right) \mathcal{H}_{n-j}^{\left(S, k_{2}-m\right)}(x, y ; \lambda, u),
\end{gathered}
$$

and

$\sum_{j=0}^{n}\left(\begin{array}{l}n \\ j\end{array}\right) \mathcal{G}_{j}^{(m)}\left(-\frac{\lambda}{u}\right) \mathcal{H}_{n-j}^{\left(S, k_{2}-m\right)}(x, y ; \lambda, u)=\left(\begin{array}{c}n \\ m\end{array}\right) m ! \sum_{j=0}^{n-m}\left(\begin{array}{c}n-m \\ j\end{array}\right) \mathcal{E}_{j}^{(m)}\left(-\frac{\lambda}{u}\right) \mathcal{H}_{n-m-j}^{\left(S, k_{2}-m\right)}(x, y ; \lambda, u)$.

\section{Conclusions}

In this article, we defined new families of two parametric kinds of Eulerian-type polynomials with their generating functions. Combining the Euler's formula with these generating functions, their functional equations and partial derivative equations, we investigated some properties of these polynomials. We also derived new identities and relations involving Apostol-type numbers and polynomials, the Stirling numbers, Fubini-type polynomials, combinatorial sums and also the aforementioned new families of polynomials. Thus, the aforementioned results have the potential to motivate the curious researchers and readers for future research on these special numbers and polynomials. Consequently, the results of this article may potentially be used in mathematics, in mathematical physics and in engineering.

Author Contributions: All authors contribute equally to this paper.

Funding: This research received no external funding.

Acknowledgments: The present investigation was supported by Scientific Research Project Administration of Akdeniz University. The authors would like to thank the referees for their valuable comments on the present paper.

Conflicts of Interest: The authors declare no conflict of interest.

\section{References}

1. Apostol, T. On the Lerch zeta function. Pac. J. Math. 1951, 1, 161-167. [CrossRef]

2. Bayad, A.; Kim, T. Identities for Apostol-type Frobenius-Euler polynomials resulting from the study of a nonlinear operator. Russ. J. Math. Phys. 2016, 23, 164-171. [CrossRef]

3. Cangul, I.N.; Cevik, S.; Simsek, Y. Generalization of $q$-Apostol-type Eulerian numbers and polynomials, and their interpolation functions. Adv. Stud. Contemp. Math. 2015, 25, 211-220.

4. Carlitz, L. Eulerian numbers and polynomials. Math. Mag. 1959, 32, 247-260. [CrossRef]

5. Choi, J.; Kim, D.S.; Kim, T.; Kim, Y.H. A note on some identities of Frobenius-Euler numbers and polynomials. Int. J. Math. Math. Sci. 2012, 861797, 1-9. [CrossRef]

6. Duan, R.; Shen, S. Bernoulli polynomials and their some new congruence properties. Symmetry 2019, 11, 365. [CrossRef]

7. Kilar, N.; Simsek, Y. A new family of Fubini type numbers and polynomials associated with Apostol-Bernoulli numbers and polynomials. J. Korean Math. Soc. 2017, 54, 1605-1621.

8. Kilar, N.; Simsek, Y. Relations on Bernoulli and Euler polynomials related to trigonometric functions. Adv. Stud. Contemp. Math. 2019, 29, 191-198.

9. Kilar, N.; Simsek, Y. Some classes of generating functions for generalized Hermite- and Chebyshev-type polynomials: Analysis of Euler's formula. arXiv 2019, arxiv:1907.03640.

10. Kim, D.S.; Kim, T. Some new identities of Frobenius-Euler numbers and polynomials. J. Inequal. Appl. 2012, 307, 1-10. [CrossRef] 
11. Kim, T.; Ryoo, C.S. Some identities for Euler and Bernoulli polynomials and their zeros. Axioms 2018, 7, 56. [CrossRef]

12. Kucukoglu, I.; Simsek, Y. Identities and relations on the $q$-Apostol type Frobenius-Euler numbers and polynomials. J. Korean Math. Soc. 2019, 56, 265-284.

13. Kurt, B.; Simsek, Y. On the generalized Apostol-type Frobenius-Euler polynomials. Adv. Differ. Equ. 2013, 1, 1-9. [CrossRef]

14. Luo, Q.-M. Apostol-Euler polynomials of higher order and Gaussian hypergeometric functions. Taiwan. J. Math. 2006, 10, 917-925. [CrossRef]

15. Luo Q.-M; Srivastava H.M. Some generalizations of the Apostol-Genocchi polynomials and the Stirling numbers of the second kind. Appl. Math. Comput. 2011, 217, 5702-5728.

16. Masjed-Jamei, M.; Koepf, W. Symbolic computation of some power-trigonometric series. J. Symb. Comput. 2017, 80, 273-284. [CrossRef]

17. Simsek, Y. Complete sum of products of $(h ; q)$-extension of Euler polynomials and numbers. J. Differ. Equ. Appl. 2010, 16, 1331-1348. [CrossRef]

18. Simsek, Y. Generating functions for $q$-Apostol type Frobenius-Euler numbers and polynomials. Axioms 2012, 1, 395-403. [CrossRef]

19. Simsek, Y. Generating functions for generalized Stirling type numbers, Array type polynomials, Eulerian type polynomials and their applications. Fixed Point Theory Appl. 2013, 87, 1-28. [CrossRef]

20. Simsek, Y. Generating functions for finite sums involving higher powers of binomial coefficients: Analysis of hypergeometric functions including new families of polynomials and numbers. J. Math. Anal. Appl. 2019, 477, 1328-1352. [CrossRef]

21. Srivastava, H.M. Some generalizations and basic (or $q$-) extensions of the Bernoulli, Euler and Genocchi polynomials. Appl. Math. Inf. Sci. 2011, 5, 390-444.

22. Srivastava, H.M.; Kim, K.; Simsek, Y. q-Bernoulli numbers and polynomials associated with multiple $q$-zeta functions and basic L-series. Russ. J. Math. Phys. 2005, 12, 241-268.

23. Srivastava, H.M.; Boutiche, M.A.; Rahmani, M. Some explicit formulas for the Frobenius-Euler polynomials of higher order. Appl. Math. Inf. Sci. 2017, 11, 621-626. [CrossRef]

24. Srivastava, H.M.; Choi, J. Zeta and q-Zeta Functions and Associated Series and Integrals; Elsevier: Amsterdam, The Netherlands, 2012.

25. Srivastava, H.M.; Masjed, M.J.; Beyki, M.R. A parametric type of the Apostol-Bernoulli, Apostol-Euler and Apostol-Genocchi polynomials. Appl. Math. Inf. Sci. 2018, 12, 907-916. [CrossRef]

26. Srivastava, H.M.; Kizilates, C. A parametric kind of the Fubini-type polynomials. RACSAM 2019. [CrossRef]

27. Zhao, J.H.; Chen, Z.Y. Some symmetric identities involving Fubini polynomials and Euler numbers. Symmetry 2018, 10, 303.

(C) 2019 by the authors. Licensee MDPI, Basel, Switzerland. This article is an open access article distributed under the terms and conditions of the Creative Commons Attribution (CC BY) license (http://creativecommons.org/licenses/by/4.0/). 